\title{
On the simple calculation of walking efficiency without kinematic information for its convenient use
}

\author{
Daijiro Abe ${ }^{1 *}$, Yoshiyuki Fukuoka ${ }^{2}$ and Masahiro Horiuchi ${ }^{3}$
}

\begin{abstract}
Background: Since walking is a daily activity not to require the maximal effort in healthy populations, a very few universal bio-parameters and/or methods have been defined to evaluate individual walking characteristics in those populations. A concept of "economy" is a potential candidate; however, walking economy highly depends on speed, so direct comparisons of economy values are difficult between studies. We investigated whether the vertical component of net walking "efficiency" (Eff vert; \%) is constant across speed. In that case, direct comparisons of Eff vert will be possible between studies or individuals at any voluntary speed.

Methods: Thirty young male participants walked at eight speeds on the level or $\pm 5 \%$ gradients, providing vertical speeds $\left(v_{\text {vert }}\right)$. Differences in energy expenditure between level and uphill or downhill gradients $(\Delta \mathrm{EE})$ were calculated. The metabolic rate for vertical component $\left(\mathrm{MR}_{\text {vert }}\right)$ was calculated by multiplying $\Delta \mathrm{EE}$ with body mass (BM). The mechanical power output for vertical component $\left(P_{\text {mech }}\right)$ was calculated by multiplying BM, gravitational acceleration, and $v_{\text {vert. }}$. Eff vert was obtained from the ratio of $P_{\text {mech }}$ to $M R_{\text {vert }}$ at each $v_{\text {vert. }}$. Delta efficiency (Delta-E; \%) was also calculated from the inverse slope of the regression line representing the relationship of $P_{\text {mech }}$ to $M R_{\text {vert }}$.

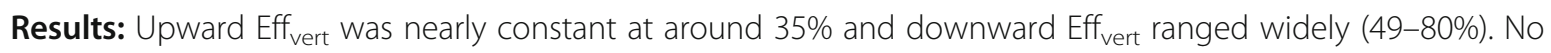
significant differences were observed between upward Delta-E (35.5 $\pm 8.8 \%)$ and Eff vert at any speeds, but not between downward Delta-E (44.9 $\pm 12.8 \%)$ and Eff vert.

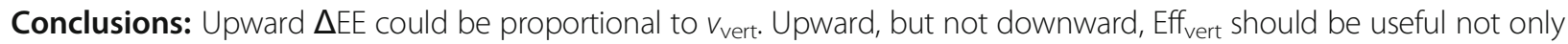
for healthy populations but also for clinical patients to evaluate individual gait characteristics, because it requires only two metabolic measurements on the level and uphill gradients without kinematic information at any voluntary speed.

Trial registration: UMIN000017690 (R000020501; registered May 26th, 2015, before the first trial) and UMIN000031456 (R000035911; registered Feb. 23rd, 2018, before the first trial).
\end{abstract}

Keywords: Bipedalism, Locomotion, Gait, Model analysis

\section{Background}

Walking and running are two of the major gait patterns in the erect bipedal locomotion. Previous literatures have evaluated individual running capacities using already established physiological parameters. However, except for race walking, walking is a daily activity not to require individual maximal effort, so relatively fewer biomechanical parameters or methods have been developed to

\footnotetext{
* Correspondence: abed@ip.kyusan-u.ac.jp

${ }^{1}$ Biodynamics Laboratory, Center for Health and Sports Science, Kyushu Sangyo University, 2-3-1 Matsukadai, Higashi-ku, Fukuoka City, Fukuoka 813-8503, Japan

Full list of author information is available at the end of the article
}

evaluate individual walking characteristics in healthy populations and clinical patients [1]. A concept of "efficiency" is a potential and universal candidate; however, the evaluation of walking efficiency takes considerable technical effort because of the need to process kinematic information using expensive devices and specialized software $[1,2]$. Nevertheless, it still involves various uncertainties in the quantification of the mechanical work done by multiple body segments (internal work), as well as the "negative" work [2]. Thus, literatures often use the concept of "economy," the energy cost of transport per unit distance $\left(\mathrm{CoT} ; \mathrm{J} \cdot \mathrm{kg}^{-1} \cdot \mathrm{km}^{-1}\right)$, because this only requires metabolic information [3]. However, walking 
economy is highly dependent on speed, with a U-shaped relationship between CoT values and walking speed [4-7]. Thus, as far as we know, the economical speed could be the only index for evaluating walking ability in each individual [4-11]. However, it is difficult to measure the entire CoT-speed curve for people with poor physical fitness, such as patients after surgery [8], elderly populations $[7,9,10]$, and pregnant women [11], for safety considerations. This may limit the number of metabolic measurements only at slower speeds. Differences in walking speed also make it difficult to directly compare economy values between studies.

For a given walking speed, whole-body energy expenditure $\left(\mathrm{EE} ; \mathrm{J} \cdot \mathrm{kg}^{-1} \cdot \mathrm{s}^{-1}\right.$ ) differs between the level and uphill/ downhill gradients $\left(\Delta \mathrm{EE} ; \mathrm{J} \cdot \mathrm{kg}^{-1} \cdot \mathrm{s}^{-1}\right)$; thus, the vertical component of metabolic rate $\left(\mathrm{MR}_{\text {vert }}\right.$; watt $)$ can be obtained by multiplying $\triangle \mathrm{EE}$ with body mass (BM; $\mathrm{kg}$ ). The vertical component of mechanical power output ( $P_{\text {mech }}$; watt $)$ can be calculated by multiplying BM, gravitational acceleration $\left(g ; \mathrm{m} \cdot \mathrm{s}^{-2}\right)$, and the vertical component of walking speed $\left(v_{\mathrm{ver}} ; \mathrm{m} \cdot \mathrm{s}^{-1}\right)$ [12-16]. The ratio of the $P_{\text {mech }}$ to $\mathrm{MR}_{\text {vert }}$ gives the vertical component of net walking efficiency $\left(\mathrm{Eff}_{\text {vert }} ; \%\right)$. Here, Eff $\mathrm{vert}_{\text {vach }}$ at eacheed will be possible to calculate without kinematic information.

The net "horizontal" walking efficiency calculated from both metabolic and kinematic information has varied widely between literatures (25-40\%) [17-20]. It has also been reported to have an inverse U-shaped profile as a function of horizontal walking speed $[18,20]$. In contrast, Eff $_{\text {vert }}$ as a function of speed has been estimated to be constant for each individual during uphill running [21] and climbing ergometer exercise [22]. This is because the EE required to lift the body was proportional to the gradient when walking at a given speed [23]. If Eff $_{\text {vert }}$ is constant across speeds, then an assessment of Eff $_{\text {vert }}$ requires only two metabolic measurements while walking at any voluntary speed along the level and a gentle uphill gradient. Indeed, several previous studies have used Eff $f_{\text {vert }}$ to evaluate effects of maximal strength training on gait characteristics in elderly cardiorespiratory patients $[13,14]$, schizophrenia patients with gait disturbance [12], and healthy elderly populations [16]. The metabolic measurement in those studies was always conducted at a particular speed $\left(1.0 \mathrm{~m} \cdot \mathrm{s}^{-1}\right)$; however, only one study measured healthy young populations [16], suggesting that we merely know about a potential usage of Eff ${ }_{\text {vert }}$ for other populations, particularly at faster walking speeds.

Delta efficiency (Delta-E; \%), defined as the inverse slope of the regression line representing the relationship of mechanical power output to energy consumption rate, might also be useful for evaluating walking efficiency "without" kinematic information. Although
Delta-E has been used for running in previous studies (e.g., [21]), it still requires several sets of metabolic measurements. There has been limited information for evaluating both $\mathrm{Eff}_{\text {vert }}$ and Delta-E for walking [24, $25]$. For an individual, there is only one Delta-E value for each gradient, so it is impossible to evaluate whether Eff $_{\text {vert }}$ is dependent on $v_{\text {vert }}$. It is interesting to note that downhill Eff vert $_{\text {gradually decreases as a }}$ function of walking speed [25]. This could be due to changes in the recovery rate of pendular energy transduction between kinetic energy and gravitational potential energy $[2,25,26]$ and greater eccentric muscle contractions during downhill walking, being associated with the utilization of stored elastic energy $[19,20$, 25]. Contrary to downhill walking, uphill walking makes us more exhausted compared to level walking, because more "positive" work in the exercising muscles is necessary during uphill walking than level or downhill walking [25]. We hypothesized that upward, but not downward, Eff ${ }_{\text {vert }}$ values would be constant across various $v_{\text {vert }}$. To test this hypothesis and to expand a potential procedure of previous studies [12-16, 21, 23, 24], the aim of this study was to obtain $\mathrm{Eff}_{\text {vert }}$ and Delta-E values for a range of $v_{\text {vert }}$ in healthy young participants without kinematic information.

\section{Methods \\ Participants}

As shown in Fig. 1, this study used additional data from already published investigations [4-6] from an entirely different perspective. Since several participants were overlapped among those investigations, the data from the first measurement in those overlapped participants were used in this study. Thirty males were involved in the level and uphill conditions with a mean age of $19.9 \pm 1.0$ years, stature of $170.5 \pm 5.7$ $\mathrm{cm}$, and body mass of $59.8 \pm 6.8 \mathrm{~kg}$, respectively (mean \pm standard deviation [SD]). Downhill gradient $(-5 \%)$ was not tested in one of the previous studies [6], so 20 of the 30 males $(20.0 \pm 1.1$ years, $170.0 \pm$ $6.3 \mathrm{~cm}$, and $59.8 \pm 5.5 \mathrm{~kg}$ ) were involved in a downhill condition. An ethical committee of Kyushu Sangyo University approved all procedures of this study (H240324, H27-0002, and H28-0001).

\section{Study procedures}

Exercise protocols were also described in Fig. 1. All participants continuously walked on a motor-driven treadmill (LABORDO LXE1200, Senoh, Japan) at eight horizontal walking speeds from 0.667 to $2.028 \mathrm{~m} \mathrm{~s}^{-1}$ on the level $( \pm 0 \%)$ and uphill $\left(+5 \%=+2.862^{\circ}\right)$ gradients. These horizontal speeds and gradients provided $v_{\text {vert }}$ from 0.033 to $0.101 \mathrm{~m} \mathrm{~s}^{-1}$ calculated from a following equation [13-16]. 


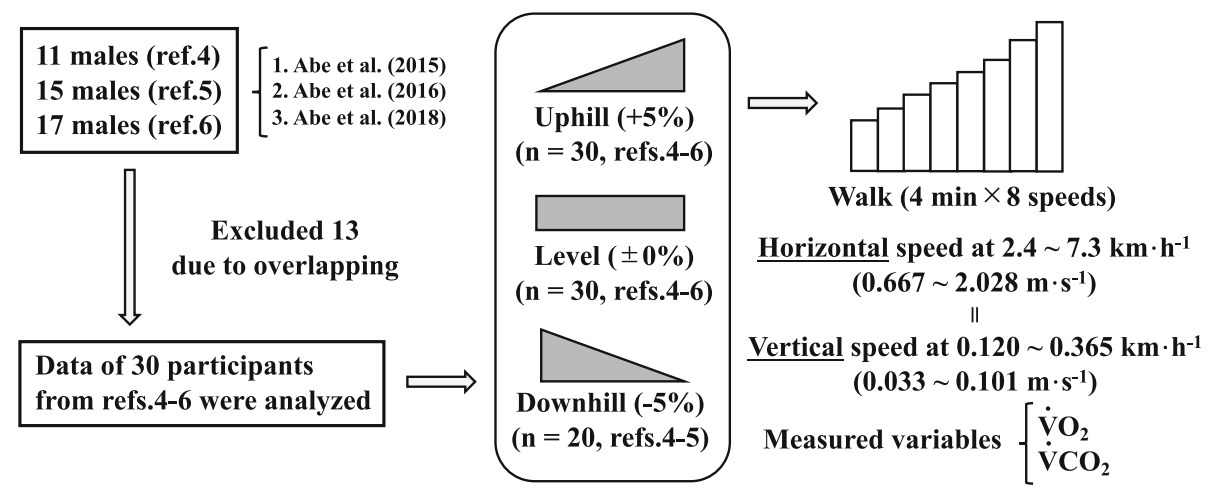

Fig. 1 Number of participants on each gradient and measured conditions were schematically illustrated at eight walking speeds

$v_{\text {vert }}=$ each walking speed $\cdot \sin \left(2.862^{\circ}\right)$

\section{Measurements and analysis}

Oxygen uptake $\left(\mathrm{VO}_{2} ; \mathrm{mL} \cdot \mathrm{kg}^{-1} \cdot \mathrm{min}^{-1}\right)$ and carbon dioxide output $\left(\mathrm{VCO}_{2} ; \mathrm{mL} \cdot \mathrm{kg}^{-1} \cdot \mathrm{min}^{-1}\right)$ were continuously measured for $4 \mathrm{~min}$ at each speed with a breath-bybreath system (Additional file 1: Figure S1) (AE-310S, Minato Ltd, Japan). The average of $\mathrm{VO}_{2}$ and $\mathrm{VCO}_{2}$ for the final 2 min at each speed was used to calculate the energy expenditure (EE; $\mathrm{J} \cdot \mathrm{kg}^{-1} \cdot \mathrm{s}^{-1}$ ) as follows [27].

$$
\mathrm{EE}=\frac{4.186 \cdot\left(3.869 \cdot V \mathrm{O}_{2}+1.195 \cdot V C \mathrm{O}_{2}\right)}{60}
$$

$\Delta \mathrm{EE}\left(\mathrm{J} \cdot \mathrm{kg}^{-1} \cdot \mathrm{s}^{-1}\right)$ at each $v_{\text {vert }}$ was multiplied by BM to obtain the $\mathrm{MR}_{\text {vert }}$ :

$$
\mathrm{MR}_{\mathrm{vert}}=\Delta \mathrm{EE} \cdot \mathrm{BM}
$$

$P_{\text {mech }}$ was obtained by multiplying the BM, $g$, and $v_{\text {vert }}[12-16,21]$ :

$$
P_{\text {mech }}=\mathrm{BM} \cdot g \cdot v_{\text {vert }}
$$

$\mathrm{Eff}_{\text {vert }}$ is given by the following equation. Because $\Delta \mathrm{EE}$ was obtained by a subtraction between different gradients, the calculated Eff ${ }_{\text {vert }}$ was the net value.

$$
\mathrm{Eff}_{\mathrm{vert}}=\frac{P_{\text {mech }}}{M R_{\text {vert }}} \cdot 100
$$

We also calculated the relationship between $P_{\text {mech }}$ and $\mathrm{MR}_{\text {vert }}$ across eight measured $v_{\text {vert }}$ for each participant on both gradients as follows:

$$
\mathrm{MR}_{\mathrm{vert}}=a \cdot \mathrm{P}_{\mathrm{mech}}+b
$$

where $a$ and $b$ are constants. Delta-E is the inverse slope of the regression line given in Eq. 6 [23, 24], so it can be calculated as follows:

$$
\text { Delta-E }=\frac{1}{\alpha} \cdot 100
$$

Equation 7 means that each individual has only one Delta-E value for each gradient.

\section{Statistical analysis}

The efficiency values were compared with one-way repeated measures ANOVA within participants on each gradient. When a significant $F$ value was obtained, it was examined by Bonferroni/Dunn's post hoc test. Statistical significance was accepted at $p<0.05$.

\section{Results}

Upward Eff ${ }_{\text {vert }}$ values (range, 34.1-39.9\%) did not differ significantly across measured $v_{\text {vert }}(F=1.045, p=0.403$; Fig. 2a and Additional file 2: Table S1). Downward Eff vert values (range, 48.5-79.6\%) were significantly higher at slower $v_{\text {vert }}$ than at faster $v_{\text {vert }}(F=5.116, p<0.001$; Fig. $2 \mathrm{~b}$ and Additional file 2: Table S1). Upward and downward Delta-E values were $35.5 \pm 8.8 \%$ and $44.9 \pm 12.8 \%$, respectively (Fig. 3). There were significant differences between downward Delta-E and Eff vert (Fig. 2b), but not



\section{Discussion}

A striking finding of our study was that upward $\mathrm{Eff}_{\text {vert }}$ values did not vary significantly across the range of measured $v_{\text {vert }}$ (Fig. 2a). However, downward Eff vert values were significantly higher at slower $v_{\text {vert }}$ than at faster $v_{\text {vert }}$ (Fig. 2b). These results fully supported our hypothesis. The results indicated that $\triangle \mathrm{EE}$ for the upward direction was proportional to $v_{\text {vert }}$. That is, $\Delta \mathrm{EE}$ for the upward direction can directly be explained by the amount of active muscles generating the accelerating force. This interpretation is consistent with those of some previous studies $[18,21,22]$. Because Eff $f_{\text {vert }}$ is a "dimensionless" value (\%), it can directly be compared with the results of other studies, even if measured speed and/or gradient were different. 


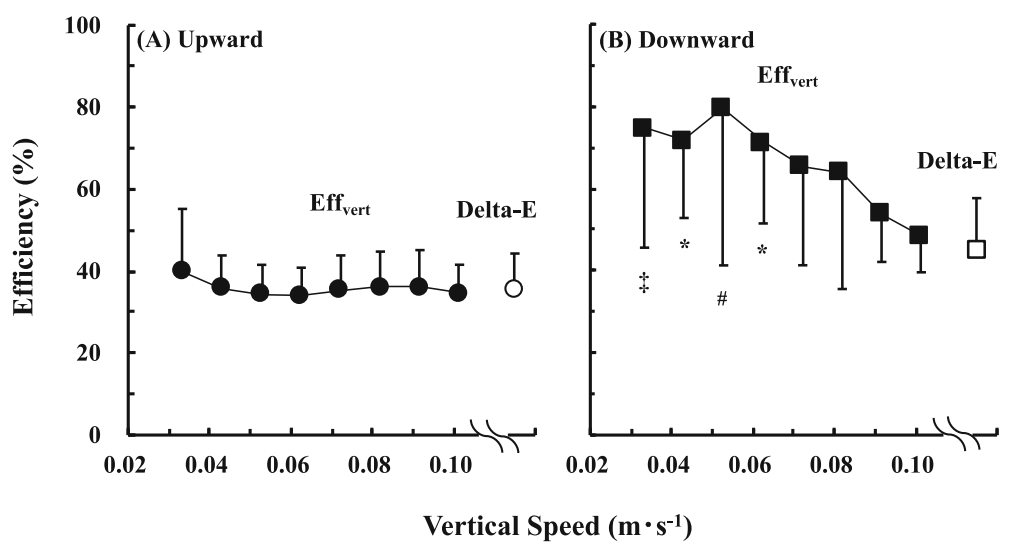

Fig. 2 Upward (a) and downward (b) Eff ${ }_{\text {vert }}$ respectively. ${ }^{*}$ indicates $p<0.05$ against $0.092,0.101 \mathrm{~m} \mathrm{~s}^{-1}$ and Delta-E. * indicates $p<0.05$ against Delta-E. ${ }^{\#}$ indicates $p<0.05$ against $0.092,0.101 \mathrm{~m} \mathrm{~s}^{-1}$, and Delta-E. Data are mean \pm standard deviation (SD)

On the basis of these interpretations, participant's attribution could not be a limitation. For example, upward Eff vert $_{\text {. }}$ was significantly improved in schizophrenia patients with gait disturbance by $19.7 \%$ after 8 -week maximal strength training [12]. This percent increase in Eff ${ }_{\text {vert }}$ is not surprising, because more considerable improvement was observed in healthy elderly populations [16] and cardiorespiratory patients $[13,15]$ after that training. A possible mechanism has been reported to be a high level of stress on all motor units including muscle activation

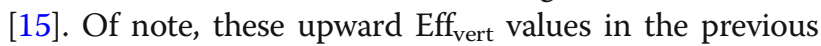
studies were evaluated only at $1.00 \mathrm{~m} \mathrm{~s}^{-1}[12-16]$.

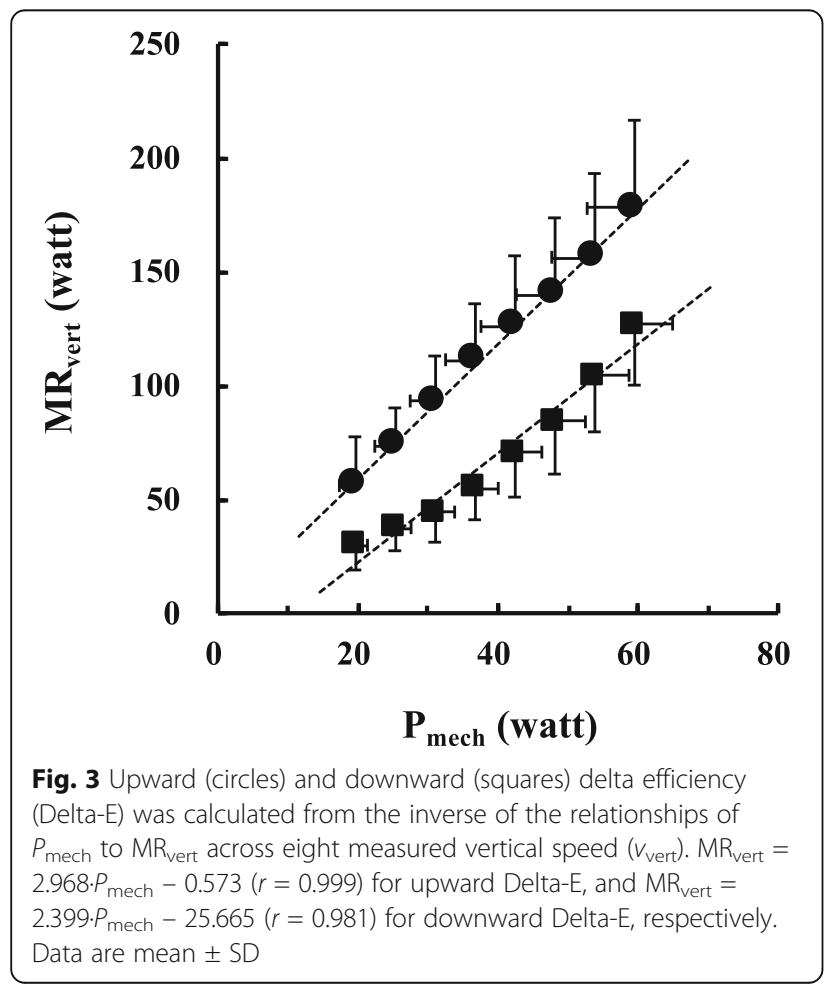

As far as we know, economical speed (ES) has been the most potential index to evaluate individual walking "ability," however, it is necessary to obtain the ES using 5-8 sets of metabolic measurements at various speeds [4-8, 11]. Each set of metabolic measurement requires $4-5 \mathrm{~min}$, so the participants need to walk at least for more than 20 min. This could not be a heavy exercise for healthy young participants; however, it may be somewhat heavy for physically poor people, such as prosthetic pedestrians, patients after surgery, obese people, pregnant women, and elderly people to execute the whole sets of metabolic measure-

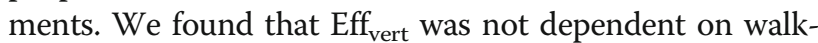
ing speeds, at least from 0.667 to $2.028 \mathrm{~m} \mathrm{~s}^{-1}$ on the uphill gradient (Fig. 2a), indicating that it can be evaluated at any designated speed. Furthermore, only two sets of metabolic measurements on the level and shallow uphill gradients without kinematic information are required. This could be a significant reduction of participants' physical strain during metabolic measurement.

It is worth noting that upward Delta-E value was not significantly different from upward $\mathrm{Eff}_{\text {vert }}$ values at any speed (Fig. 2a). Methodological considerations should be necessary. Previous studies fixed the horizontal walking speed at $1.2-1.3 \mathrm{~m} \mathrm{~s}^{-1}$ and changed treadmill gradient incrementally $[23,24]$. Conversely, we fixed the treadmill gradient at $\pm 5 \%$, and varied the walking speed incrementally. The pendular energy transduction between kinetic energy and gravitational potential energy became maximal at around $1.4 \mathrm{~m} \mathrm{~s}^{-1}[28,29]$. The minimum of the U-shaped relationship between $\mathrm{CoT}$ and horizontal walking speed occurs at around $1.4 \mathrm{~m} \mathrm{~s}^{-1}$, irrespective of the gradient [4-6]. These previous findings suggest that the grade incremental protocol may underestimate the EE ( $\fallingdotseq$ higher Delta-E). However, Delta-E reflects the inverse of an increasing rate of mechanical power output to energy consumption rate, so it may not be influenced by walking speed. Indeed, upward Delta-E of $35.5 \pm 8.8 \%$ 
was in good agreement with the result of a previous study in young adults involving both genders [23]. Given these considerations, our speed incremental protocol would be equivalent to the grade incremental protocol. We need to remind that Delta-E always requires the inverse "slope" of the regression line of $\mathrm{MR}_{\text {vert }}$ to $P_{\text {mech }}$ (Eq. 6). That is, a series of metabolic measurements are necessary to obtain the "slope" from which it is calculated, so it is practically available only for fit populations, but not for clinical patients.

A trend for significantly higher downward Eff $_{\text {vert }}$ values at slower $v_{\text {vert }}$ than at faster $v_{\text {vert }}$ (Fig. $2 \mathrm{~b}$ and Additional file 2: Table S1) was consistent with a previous result [25], indicating that the amount of active muscles needed to generate the accelerating force could not be proportional to $v_{\text {vert }}$ during downhill walking. Indeed, "negative" work rather than "positive" work becomes dominant during downhill walking $[25,26]$. The recovery rate of pendular energy transduction gradually decreased at faster $v_{\text {vert }}$ [26]. This individual variation of the recovery rate of the pendular energy transduction is associated with the muscular EE during negative and positive work. Indeed, the EE of negative work is one third of that of "positive" work [30]. Stored elastic energy in the Achilles tendon and gastrocnemius medialis can be utilized even during level walking at $0.75 \mathrm{~m} \mathrm{~s}^{-1}$ [20]. These interactions would be expected to vary considerably between individuals during downhill walking, given that relatively greater variations were observed in downward Eff ${ }_{\text {vert }}$ values than in upward Eff ${ }_{\text {vert }}$ values (Fig. 2b).

\section{Conclusions}

It is possible to evaluate vertical component of walking efficiency without kinematic information. Upward Eff ${ }_{\text {vert }}$ values were nearly constant across a wide range of $v_{\text {vert }}$, suggesting that EE to lift the body could be proportional to $v_{\text {vert }}$. Therefore, upward Eff $_{\text {vert }}$ should be useful for people with poor physical fitness to evaluate their gait characteristics. This is because only two metabolic measurements are required to obtain individual upward Effvert on the level and uphill gradients at any voluntary speed. However, this interpretation could not be applied to downhill walking. Delta-E was compatible with upward $\mathrm{Eff}_{\text {vert }}$, but not with most of the downward Eff $\mathrm{vert}_{\text {. }}$.

\section{Supplementary information}

Supplementary information accompanies this paper at https://doi.org/10. 1186/s40101-019-0211-4.

Additional file 1: Figure S1. Relationships between cardiorespiratory responses and walking speed at different gradients.

Additional file 2: Table S1. Summary of measured and calculated variables at each speed. $P_{\text {mechi }}$ mechanical power output for vertical directions, $\Delta \mathrm{EE}$; differences of energy expenditure between uphill and level gradients or between level and downhill gradients, $M R_{\text {vert }}$ metabolic rate for vertical directions, and Eff verti vertical efficiency. Values are mean $( \pm S D)$.

\section{Abbreviations}

ANOVA: Analysis of variance; BM: Body mass; CoT: Energy cost of transport per unit distance; Delta-E: Delta efficiency; EE: Whole-body energy expenditure; Eff vert: Vertical component of net walking efficiency;" $M R_{\text {vert }}$ V Vertical component of metabolic rate; $P_{\text {mech }}$ : Vertical component of mechanical power output; $\mathrm{VCO}_{2}$ : Carbon dioxide output; $\mathrm{VO}_{2}$ : Oxygen uptake; $v_{\text {vert }}$ : Vertical component of walking speed; $\Delta \mathrm{EE}$ : Difference of $\mathrm{EE}$ between the level and uphill/downhill gradients

\section{Acknowledgements}

We especially thank Dr. Herman Pontzer (Duke University) for providing us a noble viewpoint on the basis of "climbing efficiency" to start up this study.

\section{Authors' contributions}

$\mathrm{DA}, \mathrm{YF}$, and $\mathrm{MH}$ designed the study and interpreted the results. DA performed measurements and data analysis and prepared the tables, figures, and first manuscript. YF and $\mathrm{MH}$ revised the manuscript. All authors have read and approved the final manuscript.

\section{Funding}

This study was supported in part by Grant-in-Aid for Scientific Research from the Japan Society for the Promotion of Science (26440266JP and 19 K11541 to DA, 26650175JP to YF, 18K11012JP and 26440268JP to MH), Japan Society of Physiological Anthropology Research Grant for Young Scientists to DA, and Grant-in-Aid for KSU Scientific Research and Encouragement of Scientists to DA (No.58).

\section{Availability of data and materials}

All data used in this study were summarized and in the supplementary information.

\section{Ethics approval and consent to participate}

In accordance with the Declaration of Helsinki, all participants were provided all information about the purposes, benefits, possible risks, and experimental protocols. A written informed consent was obtained from all participants. An ethical committee established in Kyushu Sangyo University approved all procedures of this study (H240324, H27-0002, and H28-0001).

\section{Consent for publication}

Not applicable

\section{Competing interests}

The authors declare that they have no competing interests.

\section{Author details}

${ }^{1}$ Biodynamics Laboratory, Center for Health and Sports Science, Kyushu Sangyo University, 2-3-1 Matsukadai, Higashi-ku, Fukuoka City, Fukuoka 813-8503, Japan. ${ }^{2}$ Faculty of Health and Sports Science, Doshisha University, Kyotanabe City, Kyoto, Japan. ${ }^{3}$ Division of Human Environmental Science, Mount Fuji Research Institute, Fujiyoshida City, Yamanashi, Japan.

Received: 2 April 2019 Accepted: 27 October 2019

Published online: 30 December 2019

\section{References}

1. McCamley JD, Cutler EL, Schmid KK, Wurdeman SR, Johanning JM, Pipinos II, Myers SA. Gait mechanics differences between healthy controls and patients with peripheral artery disease after adjusting for gait velocity stride length and step width. J Appl Biomech. 2019;35:19-24.

2. Cavagna GA, Kaneko M. Mechanical work and efficiency in level walking and running. J Physiol. 1977;268:467-81.

3. Pontzer H. Economy and endurance in human evolution. Curr Biol. 2017;27: R613-R21.

4. Abe D, Fukuoka Y, Horiuchi M. Economical speed and energetically optimal transition speed evaluated by gross and net oxygen cost of transport at different gradients. PLoS One. 2015;10:e0138154. 
5. Abe D, Fukuoka Y, Horiuchi M. Muscle activities during walking and running at energetically optimal transition speed under normobaric hypoxia on gradient slopes. PLoS One. 2017;12:e0173816.

6. Abe D, Fukuoka Y, Maeda T, Horiuchi M. Energy cost and lower leg muscle activities during erect bipedal locomotion under hyperoxia. J Physiol Anthropol. 2018:37:18.

7. Horiuchi M, Endo J, Horiuchi Y, Abe D. Comparisons of energy cost and economical walking speed at various gradients in healthy, active younger and older adults. J Exerc Sci Fit. 2015;13:79-85.

8. Wezenberg D, van der Woude LH, Faber WX, de Haan A, Houdijk H. Relation between aerobic capacity and walking ability in older adults with a lower-limb amputation. Arch Phys Med Rehabil. 2013;94:1714-20.

9. Ortega JD, Beck ON, Roby JM, Turney A, Kram R. Running for exercise mitigates age-related deterioration of walking economy. PLoS One. 2014;9:e113471.

10. Mian OM, Thom JM, Ardigò LP, Narici MV, Minetti AE. Metabolic cost, mechanical work, and efficiency during walking in young and older men. Acta Physiol. 2006;186:127-39.

11. Wall-Scheffler CM, Myers MJ. Reproductive costs for everyone: how female loads impact human mobility strategies. J Hum Evol. 2013;64:448-56.

12. Heggelund J, Morken G, Helgerud J, Nilsberg GE, Hoff J. Therapeutic effects of maximal strength training on walking efficiency in patients with schizophrenia - a pilot study. BMC Res Notes. 2012;5:344.

13. Hoff J, Tjønna AE, Steinshamn S, Høydal M, Richardson RS, Helgerud J. Maximal strength training of the legs in COPD: a therapy for mechanical inefficiency. Med Sci Sports Exerc. 2007;39:220-6.

14. Høydal KL, Helgerud J, Karlsen T, Støylen A, Steinshamn S, Hoff J. Patients with coronary artery- or chronic obstructive pulmonary disease walk with mechanical inefficiency. Scand Cardiovasc J. 2007:41:405-10.

15. Karlsen T, Helgerud J, Støylen A, Lauritsen N, Hoff J. Maximal strength training restores walking mechanical efficiency in heart patients. Int J Sports Med. 2009;30:337-42.

16. Wang E, Nyberg SK, Hoff J, Zhao J, Leivseth G, Tørhaug T, Husby OS, Helgerud J, Richardson RS. Impact of maximal strength training on work efficiency and muscle fiber type in the elderly: implications for physical function and fall prevention. Exp Gerontol. 2017;91:64-71.

17. Floreani M, Rejc E, Taboga P, Ganzini A, Pišot R, Šimunič B, Biolo G, Reggiani C, Passaro A, Narici M, Rittweger J, di Prampero PE, Lazzer S. Effects of 14 days of bed rest and following physical training on metabolic cost, mechanical work, and efficiency during walking in older and young healthy males. PLoS One. 2018;13:e0194291.

18. Griffin TM, Roberts TJ, Kram R. Metabolic cost of generating muscular force in human walking: insights from load-carrying and speed experiments. J Appl Physiol. 2003;95:172-83.

19. Umberger BR, Martin PE. Mechanical power and efficiency of level walking with different stride rates. J Exp Biol. 2007;210:3255-65.

20. Farris DJ, Sawicki GS. The mechanics and energetics of human walking and running: a joint level perspective. J R Soc Interface. 2012;9:110-8.

21. Hoogkamer W, Taboga P, Kram R. Applying the cost of generating force hypothesis to uphill running. PeerJ. 2014;2:e482.

22. Hanna JD, Schmitt D, Griffin TM. The energetic cost of climbing in primates. Science. 2008;320:898.

23. Ortega JD, Farley $C T$. Effects of aging on mechanical efficiency and muscle activation during level and uphill walking. J Electromyogr Kinesiol. 2015;25:193-8.

24. Yano T. Oxygen uptake and efficiency related to external work rate during loaded walking. J Sports Med Phys Fitness. 1989:29:388-93.

25. Minetti AE, Ardigò LP, Saibene F. Mechanical determinants of gradient walking energetics in man. J Physiol. 1993;472:725-35.

26. Dewolf AH, Ivanenko YP, Lacquaniti F, Willems PA. Pendular energy transduction within the step during human walking on slopes at different speeds. PLoS One. 2017;12:e0186963.

27. Brouwer E. On simple formulae for calculating the heat expenditure and the quantities of carbohydrate and fat oxidized in metabolism of men and animals, from gaseous exchange (Oxygen intake and carbonic acid output) and urine-N. Acta Physiol Pharmacol Neerl. 1957;6:795-802.

28. Minetti AE, Capelli C, Zamparo P, di Prampero PE, Saibene F. Effects of stride frequency on mechanical power and energy expenditure of walking. Med Sci Sports Exerc. 1995;27:1194-202.

29. Willems PA, Cavagna GA, Heglund NC. External, internal and total work in human locomotion. J Exp Biol. 1995;198:379-93.

30. Aura O, Komi PV. Mechanical efficiency of pure positive and pure negative work with special reference to the work intensity. Int J Sports Med. 1986;7:44-9.

\section{Publisher's Note}

Springer Nature remains neutral with regard to jurisdictional claims in published maps and institutional affiliations.

\section{Ready to submit your research? Choose BMC and benefit from:}

- fast, convenient online submission

- thorough peer review by experienced researchers in your field

- rapid publication on acceptance

- support for research data, including large and complex data types

- gold Open Access which fosters wider collaboration and increased citations

- maximum visibility for your research: over $100 \mathrm{M}$ website views per year

At BMC, research is always in progress.

Learn more biomedcentral.com/submissions 